\title{
A COMPANION TO THE OSELEDEC MULTIPLICATIVE ERGODIC THEOREM
}

\author{
JOSEPH C. WATKINS
}

\begin{abstract}
Let $F_{1}, F_{2}, \ldots$ be a stationary sequence of continuously differentiable mappings from $[0,1]$ into the set of $d \times d$ matrices. Assume $F_{k}(0)=I$ for each $k$ and $E\left[\sup _{0 \leq p \leq 1}\left\|F_{k}^{\prime}(p)\right\|\right]<\infty$. Let I denote the invariant sigma field for the sequence. Then

$$
\operatorname{limit}_{n \rightarrow \infty} F_{n}\left(\frac{1}{n}\right) \cdots F_{2}\left(\frac{1}{n}\right) F_{1}\left(\frac{1}{n}\right)=\exp E\left[F_{1}^{\prime}(0) \mid I\right]
$$
\end{abstract}

with probability one.

Let $A$ be any $d \times d$ matrix, and let $\|A\|$ denote its norm as an operator. Choose a vector $y$. Then a simple fact from linear algebra is that the following limit exists:

$$
\chi(y, A)=\operatorname{limit}_{n \rightarrow \infty} \frac{1}{n} \log \left\|A^{n} y\right\| .
$$

Letting $y_{\lambda}$ denote any vector satisfying $A y_{\lambda}=\lambda y_{\lambda}$, and $(\cdot, \cdot)$ denote inner product, we see that, in fact, the Liapounov characteristic

$$
\chi(y, A)=\max \left\{\log |\lambda|:\left(y, y_{\lambda}\right) \neq 0\right\} .
$$

With this fact as basis, the Oseledec multiplicative ergodic theorem establishes the existence of random Liapounov characteristics

$$
\chi(y)=\operatorname{limit}_{n \rightarrow \infty} \frac{1}{n} \log \left\|X_{n} \cdots X_{2} X_{1} y\right\|
$$

for $\left\{X_{k} ; k \geq 1\right\}$ a stationary sequence of random matrices. This limit provides a description of the rate of growth of the norm for a random walk on the matrices.

If, instead, we wish to understand the ergodic behavior of an infinitesimal random walk, then we are concerned with a long product of random matrices where each matrix in the product is very nearly the identity matrix. The linear algebra identity appropriate to this situation is

$$
\operatorname{limit}_{n \rightarrow \infty} F\left(\frac{1}{n}\right)^{n}=\exp F^{\prime}(0),
$$

where $F$ is a continuously differentiable mapping from $[0,1]$ into $M(d)$, the set of $d \times d$ matrices, with $F(0)=I$. With this in mind we may easily conjecture the following theorem.

THEOREM. Let $\left\{F_{k} \in C^{1}([0,1] ; M(d)) ; k \geq 1\right\}$ be a stationary sequence having invariant sigma field $I$. Assume for each $k$ that $F_{k}(0)=I$ and that

$$
E\left[\sup _{0 \leq p \leq 1}\left\|F_{k}^{\prime}(p)\right\|\right]<\infty .
$$

Received by the editors January 17, 1985 and, in revised form, February 25, 1985.

1980 Mathematics Subject Classification (1985 Revision). Primary 22D40, 47A35, 60F15, 60G10. 
Then

$$
P\left\{\operatorname{limit}_{n \rightarrow \infty} F_{n}\left(\frac{1}{n}\right) \cdots F_{2}\left(\frac{1}{n}\right) F_{1}\left(\frac{1}{n}\right)=\exp E\left[F_{1}^{\prime}(0) \mid I\right]\right\}=1 .
$$

PROOF. Set

$$
X_{n}(t)=F_{[n t]}\left(\frac{1}{n}\right) \cdots F_{2}\left(\frac{1}{n}\right) F_{1}\left(\frac{1}{n}\right)
$$

and $X(t)=\exp t E\left[F_{1}^{\prime}(0) \mid I\right]$. Then

$$
X(t)=I+\int_{0}^{t} E\left[F_{1}^{\prime}(0) \mid I\right] X(s) d s
$$

Let $\varepsilon>0$; if we can show that

(1) $P\left\{\sup _{0 \leq t \leq 1}\left\|X_{n}(t)-I-\int_{0}^{t} E\left[F_{1}^{\prime}(0) \mid I\right] X_{n}(s) d s\right\|<\varepsilon\right.$ almost always $\}=1$,

then for all sufficiently large $n$,

$$
\begin{gathered}
\left\|X_{n}(t)-X(t)-\int_{0}^{t} E\left[F_{1}^{\prime}(0) \mid I\right]\left(X_{n}(s)-X(s)\right) d s\right\|<\varepsilon \\
\left\|X_{n}(t)-X(t)\right\|<\varepsilon+\int_{0}^{t}\left\|E\left[F_{1}^{\prime}(0) \mid I\right]\right\|\left\|X_{n}(s)-X(s)\right\| d s
\end{gathered}
$$

for all $t \in[0,1]$. Thus by Gronwall's inequality

$$
\left\|X_{n}(t)-X(t)\right\|<\varepsilon \exp t\left\|E\left[F_{1}^{\prime}(0) \mid I\right]\right\|,
$$

or, setting $t=1$,

$$
\left\|F_{n}\left(\frac{1}{n}\right) \cdots F_{2}\left(\frac{1}{n}\right) F_{1}\left(\frac{1}{n}\right)-\exp E\left[F_{1}^{\prime}(0) \mid I\right]\right\|<\varepsilon \exp \left\|E\left[F_{1}^{\prime}(0) \mid I\right]\right\|
$$

almost always with probability one. Thus, establishing (1) is sufficient to prove the theorem.

First, let $t$ be an integral multiple of $1 / n$. Then

$$
\begin{aligned}
& X_{n}(t)-I-\int_{0}^{t} E\left[F_{1}^{\prime}(0) \mid I\right] X_{n}(s) d s \\
& =\sum_{k=1}^{n t}\left(X_{n}\left(\frac{k}{n}\right)-X_{n}\left(\frac{k-1}{n}\right)\right)-\sum_{k=1}^{n t} E\left[F_{1}^{\prime}(0) \mid I\right] X_{n}\left(\frac{k-1}{n}\right) \frac{1}{n} \\
& =\sum_{k=1}^{n t}\left(\left(F_{k}\left(\frac{1}{n}\right)-I\right) X_{n}\left(\frac{k-1}{n}\right)-E\left[F_{1}^{\prime}(0) \mid I\right] X_{n}\left(\frac{k-1}{n}\right) \frac{1}{n}\right) \\
& =\sum_{k=1}^{n t}\left(\left(F_{k}\left(\frac{1}{n}\right)-I-\frac{1}{n} F_{k}^{\prime}(0)\right)+\frac{1}{n}\left(F_{k}^{\prime}(0)-E\left[F_{1}^{\prime}(0) \mid I\right]\right)\right) X_{n}\left(\frac{k-1}{n}\right) .
\end{aligned}
$$


Therefore, for any $t$,

$$
\begin{aligned}
& \left\|X_{n}(t)-I-\int_{0}^{t} E\left[F_{1}^{\prime}(0) \mid I\right] X_{n}(s) d s\right\| \\
& \leq\left(\left\|\sum_{k=1}^{[n t]}\left(F_{k}\left(\frac{1}{n}\right)-I-\frac{1}{n} F_{k}^{\prime}(0)\right)\right\|+\left\|\frac{1}{n} \sum_{k=1}^{[n t]}\left(F_{k}^{\prime}(0)-E\left[F_{1}^{\prime}(0) \mid I\right]\right)\right\|\right. \\
& \left.\quad+\left(t-\frac{[n t]}{n}\right)\left\|E\left[F_{1}^{\prime}(0) \mid I\right]\right\|\right)
\end{aligned}
$$

Therefore

$$
\begin{aligned}
\text { (2) } \sup _{0 \leq t \leq 1} & \left\|X_{n}(t)-I-\int_{0}^{t} E\left[F_{1}^{\prime}(0) \mid I\right] X_{n}(s) d s\right\| \\
\leq & \left(\sum_{k=1}^{n}\left\|F_{k}\left(\frac{1}{n}\right)-I-\frac{1}{n} F_{k}^{\prime}(0)\right\|+\sup _{0 \leq t \leq 1}\left\|\frac{1}{n} \sum_{k=1}^{[n t]}\left(F_{k}^{\prime}(0)-E\left[F_{1}^{\prime}(0) \mid I\right]\right)\right\|\right. \\
& \left.+\frac{1}{n}\left\|E\left[F_{1}^{\prime}(0) \mid I\right]\right\|\right) \\
& \times \sup _{0 \leq t \leq 1}\left\|X_{n}(t)\right\| .
\end{aligned}
$$

The proof utilizes three calculations, which we now summarize in the following three claims.

Claim 1. For all $q \in[0,1]$, $\sup _{0 \leq p \leq 1} \log ^{+}\left\|F_{1}(p)\right\| \leq q \sup _{0 \leq p \leq 1}\left\|F_{1}^{\prime}(p)\right\|$.

Noting that the claim holds if $q=0$, let us assume that the claim holds for $q \in\left[0, q_{0}\right]$. If $\left\|F_{1}\left(q_{0}\right)\right\|<1$, then there exists $\varepsilon>0$ so that $\left\|F_{1}(p)\right\|<1$ for all $p \in\left[q_{0}, q_{0}+\varepsilon\right)$. Therefore,

$$
\begin{aligned}
\sup _{0 \leq p \leq q_{0}+\varepsilon} \log ^{+}\left\|F_{1}(p)\right\| & =\sup _{0 \leq p \leq q_{0}} \log ^{+}\left\|F_{1}(p)\right\| \\
& \leq q_{0} \sup _{0 \leq p \leq 1}\left\|F_{1}^{\prime}(p)\right\| \leq\left(q_{1}+\varepsilon\right) \sup _{0 \leq p \leq 1}\left\|F_{1}^{\prime}(p)\right\| .
\end{aligned}
$$

The same conclusion holds for some $\varepsilon>0$ if $\left\|F_{1}(q)\right\|$ does not increase as $q$ increases from $q_{0}$. Finally, if $\left\|F_{1}\left(q_{0}\right)\right\| \geq 1$ and $\|F(q)\|$ is increasing, then there exists $\varepsilon>0$ so that $\|F(p)\| \geq 1$ for all $p \in\left[q_{0}, q_{0}+\varepsilon\right)$. Thus, for some $p_{0} \in(q, p)$,

$$
\begin{aligned}
\log \left\|F_{1}(p)\right\| & =\log \left\|F_{1}\left(q_{0}\right)\right\|+\left.\left(p-q_{0}\right) \frac{d}{d r} \log \left\|F_{1}(r)\right\|\right|_{r=p_{0}} \\
& =\log \left\|F_{1}\left(q_{0}\right)\right\|+\left(p-q_{0}\right)\left(\left.\frac{d}{d r}\left\|F_{1}(r)\right\|\right|_{r=p_{0}}\right) /\left\|F_{1}\left(p_{0}\right)\right\| \\
& \leq q_{0} \sup _{0 \leq p \leq 1}\left\|F_{1}^{\prime}(p)\right\|+\left(p-q_{0}\right)\left\|F_{1}^{\prime}\left(p_{0}\right)\right\| \leq p \sup _{0 \leq p \leq 1}\left\|F_{1}^{\prime}(p)\right\| .
\end{aligned}
$$

This gives the claim on $\left[0, q_{0}+\varepsilon\right)$. Because $[0,1]$ is compact, the claim holds. 
Claim 2. $n\left\|F_{1}(1 / n)-I-(1 / n) F_{1}^{\prime}(0)\right\|$ converges to zero almost surely as $n \rightarrow \infty$.

$$
n\left\|F_{1}\left(\frac{1}{n}\right)-I-\frac{1}{n} F_{1}^{\prime}(0)\right\|=\left\|n \int_{0}^{1 / n} F_{1}^{\prime}(p) d p-F_{1}^{\prime}(0)\right\|
$$

which converges to zero because $F_{1}$ is continuously differentiable.

Claim 3.

$$
\sup _{0 \leq t \leq 1}\left\|\frac{1}{n} \sum_{k=1}^{[n t]}\left(F_{k}^{\prime}(0)-E\left[F_{1}^{\prime}(0) \mid I\right]\right)\right\|
$$

converges to zero almost surely as $n \rightarrow \infty$.

Applying the ergodic theoerm to each element of the matrix, we find that $(1 / n) \sum_{k=1}^{n} F_{k}^{\prime}(0)$ converges almost surely and in $L^{1}$ to some random variable $\bar{F}^{\prime}(0)$. Clearly, $\bar{F}^{\prime}(0)$ is $I$-measurable and if $D \in I$,

$$
E\left[\frac{1}{n} \sum_{k=1}^{n} F_{k}^{\prime}(0) I_{D}\right]=E\left[F_{1}^{\prime}(0) I_{D}\right]
$$

Because the convergence is in $L^{1}, E\left[\bar{F}^{\prime}(0) I_{D}\right]=E\left[F_{1}^{\prime}(0) I_{D}\right]$, and, thus $\bar{F}^{\prime}(0)=$ $E\left[F_{1}^{\prime}(0) \mid I\right]$. Therefore

$$
\left\|\frac{1}{n} \sum_{k=1}^{n}\left(F_{k}^{\prime}(0)-E\left[F_{1}^{\prime}(0) \mid I\right]\right)\right\|
$$

converges to zero almost surely. However, for any sequence $\left\{x_{k} ; k \geq 1\right\}$,

$$
0=\operatorname{limit}_{n \rightarrow \infty} \frac{1}{n} \sum_{k=1}^{n} x_{k}=\operatorname{limit}_{n \rightarrow \infty} \sup _{0 \leq t \leq 1} \frac{1}{n} \sum_{k=1}^{[n t]} x_{k}
$$

provided that either limit exists, and the third claim is substantiated.

Returning to the proof we find that

$$
\begin{aligned}
\sup _{n} \sup _{0 \leq t \leq 1} \log ^{+}\left\|X_{n}(t)\right\| & =\sup _{n} \sup _{0 \leq t \leq 1} \log ^{+}\left\|\prod_{k=1}^{[n t]} F_{k}\left(\frac{1}{n}\right)\right\| \\
& \leq \sup _{n} \sup _{0 \leq t \leq 1} \sum_{k=1}^{[n t]} \log ^{+}\left\|F_{k}\left(\frac{1}{n}\right)\right\|=\sup _{n} \sum_{k=1}^{n} \log ^{+}\left\|F_{k}\left(\frac{1}{n}\right)\right\| \\
& \leq \sup _{n} \frac{1}{n} \sum_{k=1}^{n} \sup _{0 \leq p \leq 1}\left\|F_{k}^{\prime}(p)\right\|,
\end{aligned}
$$

via the first claim. However, $\left\{\sup _{0 \leq p \leq 1}\left\|F_{k}^{\prime}(p)\right\| ; k \geq 1\right\}$ is stationary, so

$$
\frac{1}{n} \sum_{k=1}^{n} \sup _{0 \leq p \leq 1}\left\|F_{k}^{\prime}(p)\right\|
$$

converges to a random variable having mean $E\left[\sup _{0 \leq p \leq 1}\left\|F_{k}^{\prime}(p)\right\|\right]<\infty$. Therefore, $\sup _{n} \sup _{0 \leq t \leq 1}\left\|X_{n}(t)\right\|<\infty$ with probability one, and

$$
\operatorname{limit}_{M \rightarrow \infty} P\left\{\sup _{n} \sup _{0 \leq t \leq 1}\left\|X_{n}(t)\right\| \leq M\right\}=1 \text {. }
$$


Thus, we can proceed using the usual truncation argument and assume that $\sup _{n} \sup _{0 \leq t \leq 1}\left\|X_{n}(t)\right\|$ is bounded.

In light of the second claim, and in preparation for the first Borel-Cantelli lemma, choose $\left\{\varepsilon_{n} ; n \geq 1\right\}$ decreasing to zero so that

$$
P\left\{n\left\|F_{1}\left(\frac{1}{n}\right)-I-\frac{1}{n} F_{1}^{\prime}(0)\right\|>\varepsilon_{n}\right\} \leq 2^{-n}
$$

Then

$$
\begin{aligned}
\sum_{n=.}^{\infty} P & \left\{\sum_{k=1}^{n}\left\|F_{k}\left(\frac{1}{n}\right)-I-\frac{1}{n} F_{k}^{\prime}(0)\right\|>\varepsilon_{n}\right\} \\
& \leq \sum_{n=1}^{\infty} \sum_{k=1}^{n} P\left\{n\left\|F_{k}\left(\frac{1}{n}\right)-I-\frac{1}{n} F_{k}^{\prime}(0)\right\|>\varepsilon_{n}\right\} \leq \sum_{n=1}^{\infty} n 2^{-n}=2<\infty .
\end{aligned}
$$

Therefore, $\sum_{k=1}^{n}\left\|F_{k}(1 / n)-I-(1 / n) F_{k}^{\prime}(0)\right\|$ converges to zero almost surely.

The third claim coupled with the arguments above implies that the right side of inequality (2) converges to zero with probability one. This establishes (1) and with it the theorem.

REMARK. Because

$$
\left\|F_{n}\left(\frac{1}{n}\right) \cdots F_{2}\left(\frac{1}{n}\right)\right\| \leq \frac{1}{n} \sum_{k=1}^{n} \sup _{0 \leq p \leq 1}\left\|F_{k}^{\prime}(p)\right\|,
$$

and

$$
\left\{\frac{1}{n} \sum_{k=1}^{n} \sup _{0 \leq p \leq 1}\left\|F_{k}^{\prime}(p)\right\| ; n \geq 1\right\}
$$

is uniformly integrable, the convergence given in the theorem also holds in log mean, i.e.,

$$
\operatorname{limit}_{n \rightarrow \infty} E\left[\log ^{+}\left\|F_{n}\left(\frac{1}{n}\right) \cdots F_{1}\left(\frac{1}{n}\right) F_{1}\left(\frac{1}{n}\right)-\exp E\left[F_{1}^{\prime}(0) \mid I\right]\right\|\right]-0
$$

\section{REFERENCES}

1. V. I. Oseledec, A multiplicative ergodic theorem. Liapunov characteristic numbers for dymamical systems, Trans. Moscow Math. Soc. 19 (1968), 197-231.

2. Joseph C. Watkins, Limit theorems for products of random matarices: A comparison of two points of view, Proc. 1984 AMS Conf. on Random Matrices and their Products, 1985.

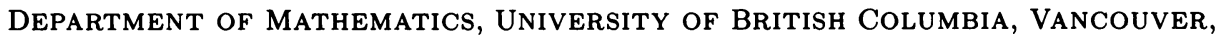
BRITISH COLUMBIA, V6T 1Y4, CANADA

Current address: Department of Mathematics, University of Southern California, University Park, Los Angeles, California 90089-1113 